\title{
BMJ Open Prognostication in critically ill patients with severe traumatic brain injury: the TBI-Prognosis multicentre feasibility study
}

\author{
Alexis F Turgeon, ${ }^{1,2}$ François Lauzier, ${ }^{1,2}$ Ryan Zarychanski, ${ }^{3}$ Dean A Fergusson, ${ }^{4}$ \\ Caroline Léger, ${ }^{1}$ Lauralyn A McIntyre, ${ }^{4,5}$ Francis Bernard, ${ }^{6}$ Andrea Rigamonti, ${ }^{7}$ \\ Karen Burns, ${ }^{7}$ Donald E Griesdale, ${ }^{8}$ Robert Green, ${ }^{9}$ Damon C Scales, ${ }^{10}$ \\ Maureen $O$ Meade, ${ }^{11}$ Martin Savard, ${ }^{12}$ Michèle Shemilt, ${ }^{1}$ Jérôme Paquet, ${ }^{13,14}$ \\ Jean-Luc Gariépy, ${ }^{14}$ André Lavoie, ${ }^{1}$ Kesh Reddy, ${ }^{15}$ Draga Jichici, ${ }^{11}$ \\ Giuseppe Pagliarello, ${ }^{5}$ David Zygun, ${ }^{16}$ Lynne Moore, ${ }^{1,17}$ for the TBI-Prognosis \\ Study Team and the Canadian Critical Care Trials Group
}

To cite: Turgeon AF, Lauzier F, Zarychanski R, et al. Prognostication in critically ill patients with severe traumatic brain injury: the TBI-Prognosis multicentre feasibility study. BMJ Open 2017;7:e013779

doi:10.1136/bmjopen-2016013779

- Prepublication history for this paper is available online. To view these files please visit the journal online (http://dx.doi.org/10.1136/ bmjopen-2016-013779).

Received 8 August 2016 Revised 8 February 2017 Accepted 10 February 2017

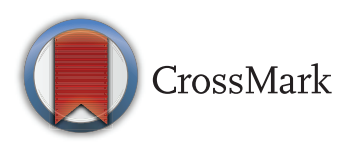

For numbered affiliations see end of article.

Correspondence to Alexis F Turgeon; alexis.turgeon@fmed.ulaval.ca

\section{ABSTRACT}

Objective: Severe traumatic brain injury is a significant cause of morbidity and mortality in young adults. Assessing long-term neurological outcome after such injury is difficult and often characterised by uncertainty. The objective of this feasibility study was to establish the feasibility of conducting a large, multicentre prospective study to develop a prognostic model of long-term neurological outcome in critically ill patients with severe traumatic brain injury.

Design: A prospective cohort study.

Setting: 9 Canadian intensive care units enrolled patients suffering from acute severe traumatic brain injury. Clinical, biological, radiological and electrophysiological data were systematically collected during the first week in the intensive care unit. Mortality and functional outcome (Glasgow Outcome Scale extended) were assessed on hospital discharge, and then 3,6 and 12 months following injury.

Outcomes: The compliance to protocolised test procedures was the primary outcome. Secondary outcomes were enrolment rate and compliance to follow-up.

Results: We successfully enrolled 50 patients over a 12 -month period. Most patients were male $(80 \%)$, with a median age of 45 years (IQR 29.0-60.0), a median Injury Severity Score of 38 (IQR 25-50) and a Glasgow Coma Scale of 6 (IQR 3-7). Mortality was 38\% (19/50) and most deaths occurred following a decision to withdraw life-sustaining therapies (18/19). The main reasons for non-enrolment were the time window for inclusion being after regular working hours $(35 \%$, $\mathrm{n}=23)$ and oversight $(24 \%, \mathrm{n}=16)$. Compliance with protocolised test procedures ranged from $92 \%$ to $100 \%$ and enrolment rate was $43 \%$. No patients were lost to follow-up at 6 months and 2 were at 12 months. Conclusions: In this multicentre prospective feasibility study, we achieved feasibility objectives pertaining to compliance to test, enrolment and follow-up.

\section{Strengths and limitations of this study}

Our study involved nine centres in five different provinces in Canada and showed the feasibility of enrolling critically ill patients with severe traumatic brain injury and assess 12 months outcome measures.

- Our study sample size allowed testing a protocol of test procedures and identifying potential pitfalls to consider for a large-scale prognostic study.

- Some eligible patients were missed due to an admission to the intensive care unit outside of working hours or were oversight by the research personnel.

We conclude that the TBI-Prognosis prospective multicentre study in severe traumatic brain injury patients in Canada is feasible.

\section{INTRODUCTION}

Severe traumatic brain injuries are catastrophic injuries primarily afflicting young individuals. ${ }^{1}$ Mortality ranges from $30 \%$ to $50 \%$, while $30 \%$ of survivors suffer from severe neurological sequelae. ${ }^{2-5}$ Given the majority of victims are young with previous excellent quality of life, substantive human, social and financial repercussions are experienced by survivors. ${ }^{6}$

With regard to victims of severe traumatic brain injury, physicians and families often face important treatment decisions. They must decide to either undertake aggressive 
care in the hope that the patient will survive with an acceptable quality of life $^{7-10}$ or to withdraw lifesustaining therapies considering an unfavourable and undesirable prognosis. Serious concerns have been expressed regarding early decisions made to withdraw life-sustaining therapies in the absence of evidencebased prognostic information. ${ }^{11-14}$ Recently, we observed significant variations in mortality and in the incidence of withdrawal of life-sustaining therapies following severe traumatic brain injury in Canada. ${ }^{8}$ Current prognostic models are of limited clinical utility as they are based on data obtained from small, ${ }^{16-18}$ single-centre ${ }^{3}{ }^{17-23}$ retrospective studies ${ }^{17} 22$ 24-26 that did not consider secondary brain injury. ${ }^{3}$ 17-21 2324 27-28 Consequently, it is not surprising to observe a wide variation in prognostic evaluation when surveying intensivists, neurosurgeons and neurologists caring for severe traumatic brain injury patients in Canada. ${ }^{29}$ The development of appropriate prognosis tools and models is necessary to help guide the decision-making process with families.

The objective of the TBI-Prognosis Feasibility Study was to assess the feasibility of conducting a large-scale, multicentre study to develop prognostic model to inform long-term prognosis in patients with severe traumatic brain injury.

\section{METHODS}

\section{Study design}

We conducted a multicentre prospective feasibility study in nine level I trauma centres across Canada. Research Ethics Board approval was obtained from each participating centre. Informed consent was obtained from surrogate decision makers prior to enrolment in most centres; deferred consent was permitted by Research Ethics Boards at two centres. This study was conducted in the Canadian healthcare system in which trauma, neurosurgery and critical care are part of a public system with universal healthcare coverage for all citizens. In Canada, major trauma care is delivered through 10 integrated provincial trauma systems. In Canada, neurocritical care is mainly delivered in combined neuro/ general intensive care units.

\section{Eligibility criteria}

We included critically ill adults ( $\geq 18$ years of age) with severe traumatic brain injury (Glasgow Coma Scale (GCS) $\leq 8$ following resuscitation) due to blunt-force trauma on day 1 of intensive care unit admission. We excluded patients anticipated to be on mechanical ventilation for $<48$ hours, patients with solid malignancy associated with a life expectancy $<12$ months, liver cirrhosis Child C, chronic heart failure (NYHA class IV), end-stage chronic respiratory disease $\left(\mathrm{O}_{2}\right.$-dependent), end-stage renal disease (chronic dialysis), previous neurological disorder with abnormal findings on radiological imaging (CT scan, MRI) or electrophysiological tests (Electroencephalogram (EEG), somatosensory evoked potentials (SSEP)) or patients who were declared brain-dead when assessed for eligibility. Patients with no fixed address were also excluded due to difficulties in conducting follow-up.

\section{Data collection}

Participants underwent a protocolised schedule of clinical, biological, radiological and electrophysiological prognostic tests or examinations. Tests and examinations used in our study were commonly utilised in the care of patients with severe traumatic brain injury for diagnostic or prognostic purposes except for blood samples. Data were collected daily from intensive care unit admission until the seventh day following the injury, death or until hospital discharge, whichever came first. These included pupillary reactivity, corneal reflex, GCS, episodes of increased intracranial hypertension $(>25 \mathrm{~mm} \mathrm{Hg})$, hypoxaemia (arterial oxygen saturation of $<90 \%$ ) and hypotension (systolic blood pressure $<90 \mathrm{~mm} \mathrm{Hg}$ ). Data were prospectively collected at the bedside using specific case report forms. We also collected serum glucose (highest and lowest value), complete blood count, INR, prothrombin time, sodium, creatinine, arterial blood gases, on a daily basis if the data were available as per clinical decision by the medical team. A schedule of prognostic biological, radiological and electrophysiological tests/ examinations was implemented (figure 1). On intensive care unit days 1, 3 and 7, CT scans were performed and blood samples were collected to measure serum biomarkers. These timelines were informed by a multicentre retrospective study and a healthcare survey of Canadian clinicians. ${ }^{8} 2930$ On intensive care unit day 7, MRI, SSEP and EEG examinations were performed. We permitted a time window of 24 hours (for CT-scan) and 48 hours (for MRI, SSEP and EEG) to reflect clinical practice and enhance feasibility over weekends.

\section{Outcome measures}

Our overarching objective of the research programme is to develop a model to predict short (discharge), mid (3 months) and long-term neurological prognosis (6 and 12 months) in patients admitted to intensive care unit with severe traumatic brain injury. The functional outcome was evaluated using the Glasgow Outcome Scale extended (GOSe) (face to face (hospitalised patients) or phone interviews (discharged patients)). ${ }^{31} 32$ Our feasibility study was designed to establish the feasibility of a large-scale study adequately powered to develop prognostic models to help inform clinical decision-making. Our primary outcome was the compliance rate to the protocolised test procedures (tests performed or not performed). We considered a $90 \%$ compliance rate to be acceptable. Secondary outcomes were enrolment rate and compliance to follow-up. We also evaluated the percentage of potentially eligible patients that were excluded, the reasons for exclusion and adverse events related to the protocol. 


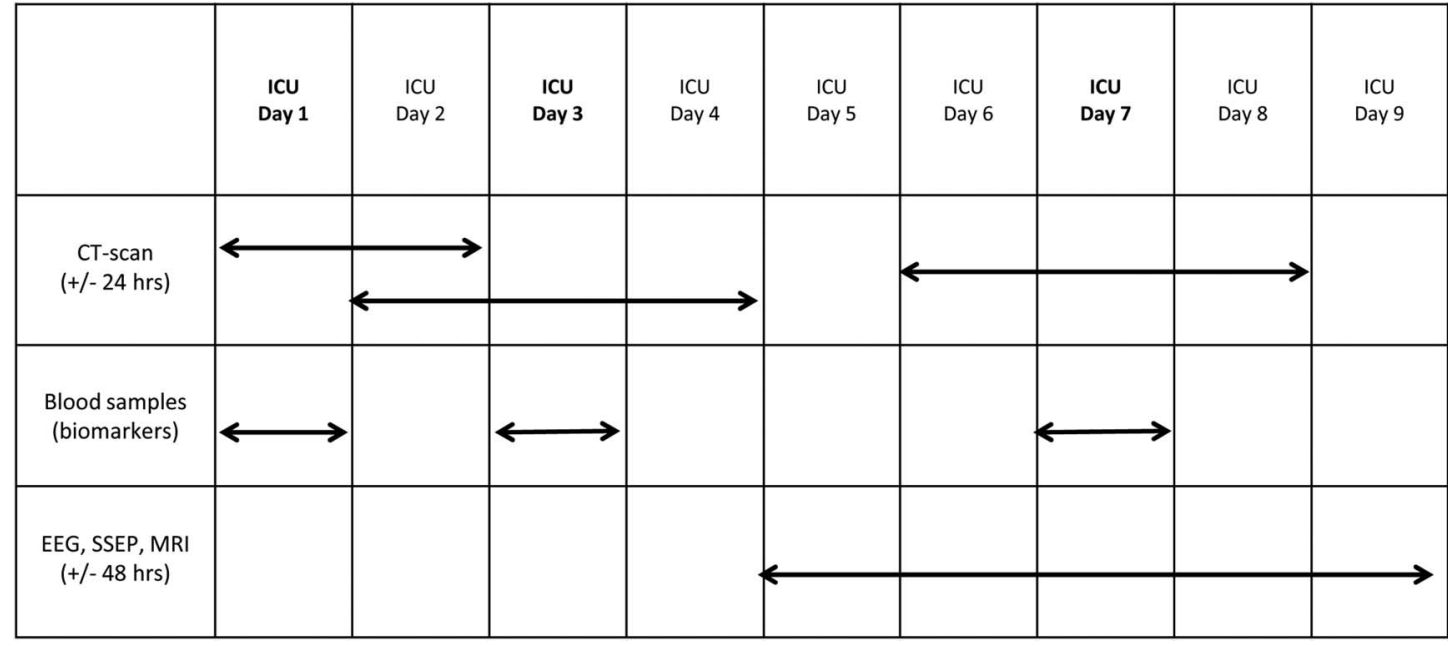

Figure 1 TBI-Prognosis test schedule. The arrows indicate the prescribed time frame to perform tests or take blood samples. The study requested that CT scans be performed on days 1, 3 and 7, with the possibility to conduct the scans 24 hours prior or after the required date. Blood samples were drawn on days 1, 3 and 7 . The EEG, SSEP and MRI tests were required on day 7 but could be obtained 48 hours before or after the seventh day. ICU, intensive care unit; SSEP, somatosensory evoked potentials. EEG: Electroencephalogram.

\section{Research team at participating centres}

At each participating centre, a research coordinator and/or research nurse was involved in the implementation of the study in the intensive care unit, daily screening, enrolment at the bedside, organisation of the schedule of tests with the attending medical team and daily data collection. Follow-ups were performed locally with face-to-face questionnaires when patients were still in hospital, or phone questionnaires, when discharged home or to another facility. Follow-ups were made during working hours for most patients.

\section{Start-up meeting}

We organised a start-up meeting using virtual technology (video conference) prior to start enrolment in the study. This starting meeting was chaired by the study manager at the coordinating centre, involved the review of the protocol, the screening, enrolment and consent process, the overall study procedure and potential pitfalls to avoid during the process.

\section{Central coordination and data monitoring}

The study was coordinated centrally by a study manager assisted by a clinical research coordinator. The study manager was responsible for supervising the implementation of the study at each site and was the primary link for the local research team to answer questions and queries during the conduction of the study. Communications through emails and phone calls to participating sites were performed on regular basis to clarify potential issues on enrolment and data collection, as well as to ascertain a close follow-up of sites. The data collection process was monitored centrally at the coordinating centre and answers queries sent to the participating centres before case report forms were considered completed. A newsletter was disseminated every other month to update centre on the enrolment in the study, but also to motivate the team and provide information on common queries and questions.

\section{Sample size}

With a sample size of 50 patients, we predicted to estimate a compliance to the scheduled test procedures of $90 \%$ with a margin of error of $10 \%$.

\section{Statistical analyses}

Descriptive statistics were used to report the data. Data on compliance to the tests procedure, enrolment rate, compliance to follow-up and overall study adherence are presented using proportions. No comparative statistical testing was performed considering the feasibility nature of this study.

\section{RESULTS}

\section{Patient enrolment}

Over a 12-month period (May 2012 to May 2013) totalising 208 weeks of active enrolment (all centres considered), participating centres screened 530 patients from which 116 were potentially eligible and 50 were enrolled $(43 \%)$. The two main reasons for non-enrolment were the time window for inclusion being after regular working hours and personnel oversight (figure 2). We observed few refusals from surrogate decision makers and physicians, as well as non-enrolment due to the absence of a surrogate decision maker. No patient, once included in the study, was excluded. One centre did not succeed to implement the study due to staffing issues and did not contribute any patients to this feasibility trial. The majority of recruitment (32 patients, 64\%) took place during weekdays; three of the centres 
Figure 2 Reasons for non-enrolment. SDM, surrogate decision maker.

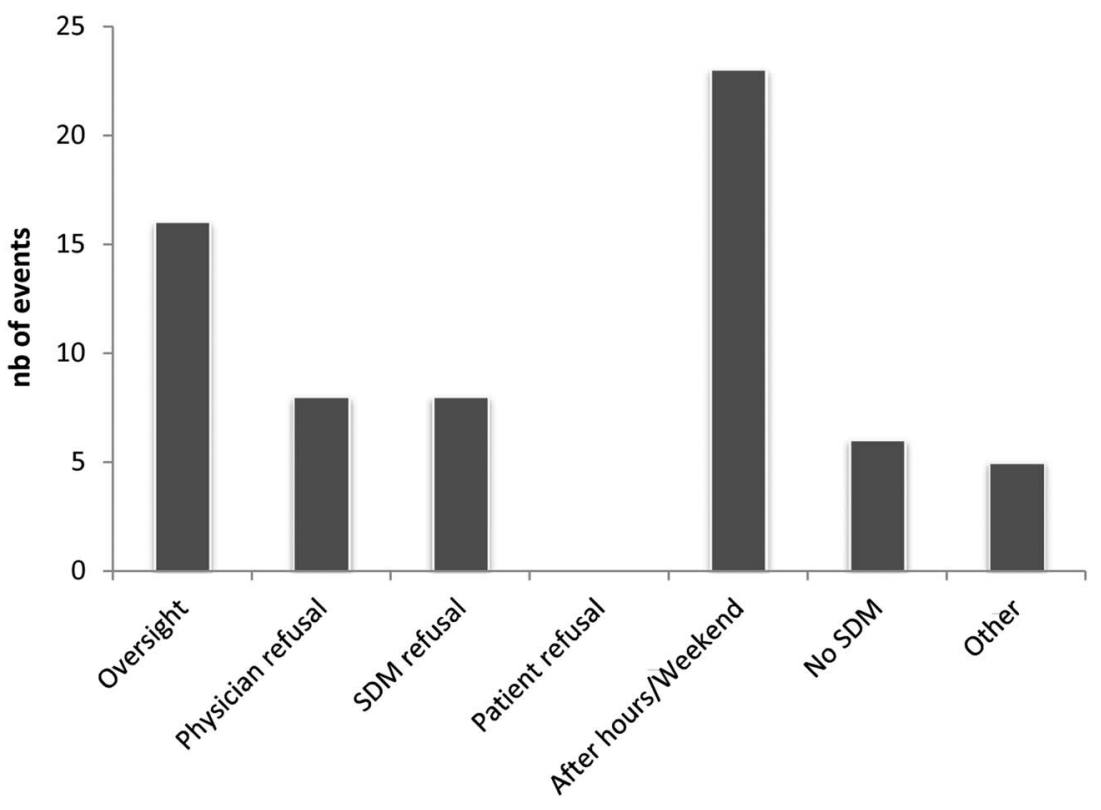

enrolled patients on weekends. Informed consent was mostly obtained (41 patients, $82 \%$ ) between 09:00 and 18:00.

\section{Patient characteristics}

The median age of participants was 45 years (IQR, 2960 years) and $80 \%$ were male (40 patients, $80 \%$ ). The median GCS at enrolment was 6 (IQR: 3-7) and the Injury Severity Score was 38 (IQR 25-50) (table 1). In $88 \%$ of patients, traumatic brain injury occurred following motor vehicle collision or fall.

\section{Compliance to the daily clinical data collection}

Clinical data for episodes of hypotension, hypoxaemia and increased intracranial pressure were successfully collected. We had three missing time points for pupillary reaction (2 patients) and one time point for the GCS (1

\section{Table 1 Patients demographic}

\begin{tabular}{lc}
\hline Characteristics & Patients (n=50) \\
\hline Age (median, IQR) & $45(29-60)$ \\
Male (n, \%) & $40(80 \%)$ \\
GCS (median, IQR) & $6(3-7)$ \\
APACHE II score (mean \pm SD) & $20.2 \pm 6.84$ \\
ISS score (median, IQR) & $38(25-30)$ \\
Absent pupillary reactivity (ICU day 1) & $36(72 \%)$ \\
Absent corneal reflex (ICU day 1) & $7(14 \%)$ \\
Cause of trauma (n, \%) & \\
MVC-occupant & $21(42 \%)$ \\
MVC-motorcyclist & $9(18 \%)$ \\
MVC-pedestrian & $2(4 \%)$ \\
Fall & $14(28 \%)$ \\
Assault & $2(4 \%)$ \\
Other & $2(4 \%)$ \\
\hline APACHE, Acute Physiology and Chronic Health Evaluation; GCS, \\
Glasgow Coma Scale; ICU, intensive care unit; ISS, Injury \\
Severity Score; MVC, motor vehicle collision.
\end{tabular}

patient). Data for the corneal reflex were however missed at least for one data point in 29 patients.

\section{Compliance to the test procedures}

The compliance to the protocol of test procedures ranged from $92 \%$ to $100 \%$, depending on the test performed. Compliance to tests was measured according to the survival status during the time window in which the test was scheduled (figure 3). We observed 94\% compliance for SSEP (3 missed tests), 96\% for EEG (2 missed tests) and $92 \%$ for MRI (4 missed tests). Day 7 MRI was delayed for $20 \% \quad(n=10)$ of the patients, most of them $(n=6)$ due to the presence of material incompatible with the performance of the MRI procedure. No CT scans were missed on days 1 and 3, while the compliance for day 7 CT scans was $96 \%$ (2 missed scans). All but one blood sample were collected (day 7); all collected blood samples were successfully shipped to the coordinating centres. The main reason for not conducting a specific test was a change in level of care (palliative care). The main explanation for performing tests outside of the time window was patient instability (haemodynamic or increased intracranial pressure). We observed no adverse events related to this study and tests performed.

\section{Follow-up of outcome measures}

Two patients were lost to follow-up at 12 months, but none were at 6 months. Overall, 33 patients $(66 \%)$ had an unfavourable outcome at 12 months (GOSe 1-4). Mortality was $38 \%(19 / 50)$ and most deaths were associated with a decision to withdraw life-sustaining therapies $(18 / 19)$. No patient died during follow-up after hospital discharge.

\section{INTERPRETATION}

In this multicentre prospective feasibility study, we achieved high compliance with the study procedures, an 
Figure 3 Compliance to the scheduled test procedures. MRI, magnetic resonance imaging; SSEP, somatosensory evoked potentials; EEG, electroencephalogram.

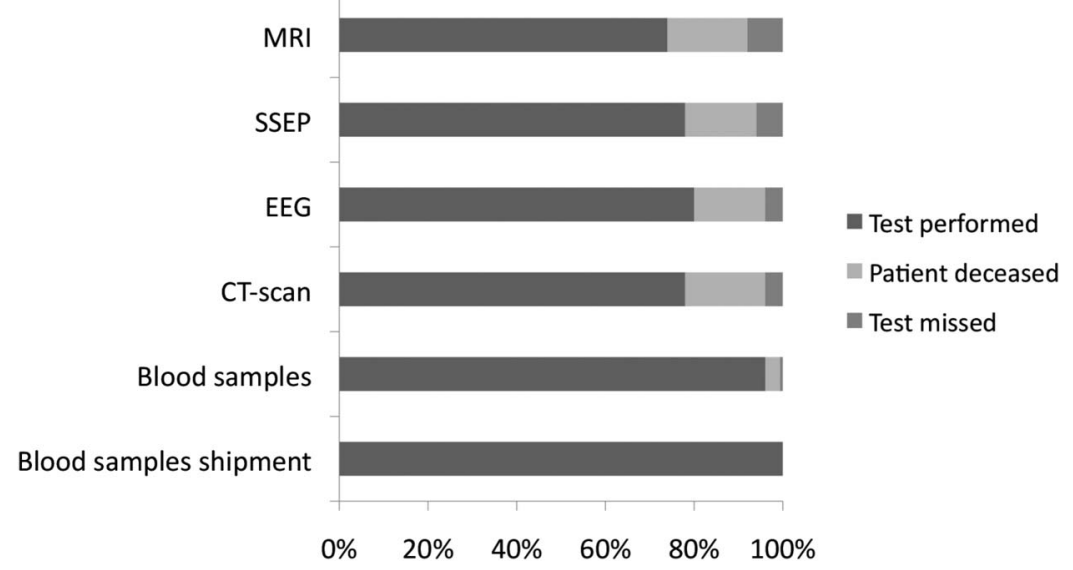

acceptable enrolment rate and had a low rate of loss to follow-up. All except one centre achieved acceptable enrolment during the study period. The lessons learnt during this multicentre feasibility prospective study have informed the design of the TBI-Prognosis multicentre prospective study (NCT02452541), which is currently ongoing.

The high compliance rate to the test procedures observed in our study is a paramount result for the feasibility of the large-scale study. Several reasons may explain this high compliance. First, our protocol is straightforward and mainly relies on reminders for timely test procedures. Second, the uniformity of the tests and the flexibility of test timing allow these tests to be included seamlessly into the patient's care continuum. Third, adherence to the test schedule has also been facilitated by local research coordinators directly interacting with the clinical personnel in the intensive care unit and championing the project, ${ }^{33}$ and by clinician guidance and enthusiasm towards the project. ${ }^{34}$ Finally, we engaged the clinical personnel working in the intensive care unit by holding information sessions describing the project and by being available to answer their queries and concerns. ${ }^{33} 35$

Pilot and feasibility studies are particularly useful in revealing study flaws and design weaknesses. ${ }^{36-38}$ In this feasibility study, we also identified some potential challenges for the conduction of the large-scale study. One of the challenges identified was the difficulty of enrolling patients admitted outside of regular working hours (evenings or week-ends). This finding that a significant proportion of patients with traumatic brain injury are admitted over the week-end was also observed in a previous cohort study of patients with mild to severe traumatic brain injury in the $\mathrm{UK}^{39}$ Owing to budgetary restrictions, but also to the available workforce, it was not always possible to have 24-hour coverage for screening and enrolment in clinical research. Using a deferred consent approach in all centres for the large-scale study is one of the avenues considered to handle this potential issue. Another important finding is our follow-up rates at 6 and 12 months that are comparable or better to the ones observed in previous large-scale multicentre trials in patients with severe traumatic brain injury. ${ }^{40} \quad 41$ Despite having missed two patients for the 12-month follow-up, we were able to follow all patients at 6 months, a result showing the possibility of not missing any patients for the large-scale study.

Following this feasibility phase of the TBI-Prognosis study, study investigators engaged with local investigators, intensive care unit nurses and research coordinators, through informal discussions and survey, to understand their experience participating in the TBI-Prognosis feasibility study. Recruitment techniques and eligibility criteria were revised and refined to improve clarity in the larger study. Deferred consent was highlighted as being especially helpful, given the time constraints and appears to be generally accepted by participants on regaining the ability to participate in the shared decision-making consent process. ${ }^{42} 43$ Indeed, the two centres that implemented this method recruited a greater number of patients than the other sites in accordance with the duration of the screening period. Strategies for approaching families in time of stress were also discussed. ${ }^{37}$ With much preparatory work completed, the TBI-Prognosis team and the Canadian Critical Care Trials Group are now undertaking the large multicentre prospective cohort study informed by the results of this pilot feasibility study.

In this multicentre prospective feasibility study, we successfully enrolled participants following an acceptable enrolment rate, reached our targeted sample size, achieved feasibility objectives pertaining to the compliance to the test procedures, compliance to follow-up, as well as the overall adherence to the study protocol. Considering our enrolment rate, we considered that 3 years will be necessary to enrol 315 patients in 17 centres across Canada in the large-scale TBI-Prognosis study. We conclude that a prospective multicentre study in severe traumatic brain injury patients in Canada aiming at developing a prognostic model in the acute phase of care is feasible. 
Author affiliations

${ }^{1}$ Population Health and Optimal Health Practices Research Unit (TraumaEmergency-Critical Care Medicine), CHU de Québec-Université Laval Research Centre, Québec City, Québec, Canada

${ }^{2}$ Department of Anesthesiology and Critical Care Medicine, Division of Critical Care Medicine, Université Laval, Québec City, Québec, Canada

${ }^{3}$ Department of Internal Medicine, Section of Critical Care and of Haematology and Medical Oncology, University of Manitoba, Winnipeg, Manitoba, Canada

${ }^{4}$ Clinical Epidemiology Unit, Center for Transfusion and Critical Care Research, Ottawa Health Research Institute, University of Ottawa, Ottawa, Ontario, Canada

${ }^{5}$ Department of Critical Care Medicine, Ottawa Hospital, University of Ottawa, Ottawa, Ontario, Canada

${ }^{6}$ Department of Medicine, Université de Montréal, Montréal, Québec, Canada

${ }^{7}$ Interdepartmental Division of Critical Care Medicine, St-Michael's Hospital, University of Toronto, Toronto, Ontario, Canada

${ }^{8}$ Department of Anesthesiology, Pharmacology and Therapeutics, University of British Columbia, Vancouver, British Columbia, Canada

${ }^{9}$ Department of Critical Care Medicine, Department of Emergency Medicine, Dalhousie University, Halifax, Nova Scotia, Canada

${ }^{10}$ Interdepartmental Division of Critical Care Medicine, Sunnybrook Hospital, University of Toronto, Toronto, Ontario, Canada

${ }^{11}$ Department of Medicine, Division of Critical Care Medicine, McMaster University, Hamilton, Ontario, Canada

${ }^{12}$ Department of Medicine, Division of Neurology, Université Laval, Québec, Québec, Canada

${ }^{13}$ Department of Surgery, Division of Neurosurgery, Université Laval, Québec, Québec, Canada

${ }^{14}$ Department Radiology and Nuclear Medicine, Université Laval, Québec, Québec, Canada

${ }^{15}$ Department of Surgery, Division of Neurosurgery, McMaster University, Hamilton, Ontario, Canada

${ }^{16}$ Department of Critical Care Medicine, University of Alberta, Edmonton, Alberta, Canada

${ }^{17}$ Department of Preventive and Social Medicine, Université Laval, Québec, Québec, Canada

Acknowledgements The authors acknowledge Ramona-Florina Fratu for her help in the statistical analyses, as well as Catherine Ouellet for assembling the data. The authors are grateful to members of the Canadian Critical Care Trials Group (CCCTG) for their support and guidance, and for CCCTG Grants and Manuscript Committee and Paule Lessard Bonaventure for the critical review of this manuscript. The authors also thank all the research coordinators and research professionals who were involved with this study for their help and dedication.

Contributors AFT, FL, RZ, DAF, LAM, FB, AR, KB, DG, RG, DCS, MOM, MS, $J P, J-L G, A L, K R, G P, D Z$ and $L M$ were involved in conception and design. AFT, FL, RZ, DAF, CL, LAM, FB, AR, KB, DG, RG, DCS, MOM, MS, MSh, JP, $J-L G, A L, K R, D J, G P, D Z$ and $L M$ were involved in the acquisition and interpretation of data. AFT, CL and MSh drafted the manuscript. AFT, FL, RZ, DAF, CL, LAM, FB, AR, KB, DG, RG, DCS, MOM, MS, MSh, JP, J-LG, AL, KR, $D J, G P, D Z$ and $L M$ were involved in revising the manuscript and approved the version published.

Funding This study was funded by the Fonds de la Recherche du QuébecSanté (FRQS) (grant \#5888) and the Canadian Intensive Care Foundation (CICF). AFT is the Canada Research Chair in Critical Care Neurology and Trauma. LM, LAM, AFT and RZ are or were recipients of New Investigator Awards from the Canadian Institutes of Health Research (CIHR) during the conduction of the study. AFT and FL are supported by the Traumatology Research Consortium of the FRQS. FL is a recipient of a salary support Award from the FRQS.

Competing interests None declared.

Ethics approval Centre Hospitalier Affilié Universitaire de Québec (CHAUQ) Research Ethics Board.

Provenance and peer review Not commissioned; externally peer reviewed.

Data sharing statement No additional data are available.
Open Access This is an Open Access article distributed in accordance with the Creative Commons Attribution Non Commercial (CC BY-NC 4.0) license, which permits others to distribute, remix, adapt, build upon this work noncommercially, and license their derivative works on different terms, provided the original work is properly cited and the use is non-commercial. See: http:// creativecommons.org/licenses/by-nc/4.0/

\section{REFERENCES}

1. National Scientific Advisory Committee. Identifying priorities for research and capacity development in injury as a multi-institute strategic initiative within the Canadian Institutes of Health Research. In: Listening for Direction on Injury: final report. Canada: Instituts canadiens de recherche en santé, 2004.

2. Medical aspects of the persistent vegetative state. The Multi-Society Task Force on PVS. N Engl J Med 1994;330:1499-508.

3. Lannoo E, Van Rietvelde F, Colardyn F, et al. Early predictors of mortality and morbidity after severe closed head injury. J Neurotrauma 2000;17:403-14.

4. Levati A, Farina ML, Vecchi G, et al. Prognosis of severe head injuries. J Neurosurg 1982;57:779-83.

5. Zygun DA, Laupland KB, Hader WJ, et al. Severe traumatic brain injury in a large Canadian health region. Can J Neurol Sci 2005;32:87-92.

6. Meerding WJ, Mulder S, van Beeck EF. Incidence and costs of injuries in The Netherlands. Eur J Public Health 2006;16:272-8.

7. Kramer AH, Zygun DA. Neurocritical care: why does it make a difference? Curr Opin Crit Care 2014;20:174-81.

8. Turgeon AF, Lauzier F, Simard JF, et al. Mortality associated with withdrawal of life-sustaining therapy for patients with severe traumatic brain injury: a Canadian multicentre cohort study. CMAJ 2011;183:1581-8.

9. van Beinum A, Hornby L, Ramsay T, et al. Exploration of withdrawal of life-sustaining therapy in Canadian intensive care units. J Intensive Care Med 2016;31:243-51.

10. Huynh TN, Walling AM, Le TX, et al. Factors associated with palliative withdrawal of mechanical ventilation and time to death after withdrawal. J Palliat Med 2013;16:1368-74.

11. Becker KJ, Baxter AB, Cohen WA, et al. Withdrawal of support in intracerebral hemorrhage may lead to self-fulfilling prophecies. Neurology 2001;56:766-72.

12. Mayer SA, Kossoff SB. Withdrawal of life support in the neurological intensive care unit. Neurology 1999;52:1602-9.

13. O'Callahan JG, Fink C, Pitts LH, et al. Withholding and withdrawing of life support from patients with severe head injury. Crit Care Med 1995;23:1567-75.

14. Muehlschlegel S, Shutter L, Col N, et al. Decision aids and shared decision-making in neurocritical care: an unmet need in our NeurolCUs. Neurocrit Care 2015;23:127-30.

15. Côte N, Turgeon AF, Lauzier F, et al. Factors associated with the withdrawal of life-sustaining therapies in patients with severe traumatic brain injury: a multicenter cohort study. Neurocrit Care 2013;18:154-60.

16. Brain Trauma Foundation, American Association of Neurological Surgeons, Joint section on neurotrauma and critical care. Management and prognosis of severe traumatic brain injury. New York, USA: Brain Trauma Foundation, 2000.

17. Lai YC, Chen FG, Goh MH, et al. Predictors of long-term outcome in severe head injury. Ann Acad Med Singapore 1998;27:326-31.

18. Rovlias A, Kotsou S. Classification and regression tree for prediction of outcome after severe head injury using simple clinical and laboratory variables. J Neurotrauma 2004;21:886-93.

19. Andrews PJ, Sleeman $\mathrm{DH}$, Statham PF, et al. Predicting recovery in patients suffering from traumatic brain injury by using admission variables and physiological data: a comparison between decision tree analysis and logistic regression. J Neurosurg 2002; 97:326-36.

20. Demetriades D, Kuncir E, Velmahos GC, et al. Outcome and prognostic factors in head injuries with an admission Glasgow Coma Scale score of 3. Arch Surg 2004;139:1066-8.

21. Fearnside MR, Cook RJ, McDougall $P$, et al. The Westmead Head Injury Project outcome in severe head injury. A comparative analysis of pre-hospital, clinical and CT variables. $\mathrm{Br} J$ Neurosurg 1993;7:267-79.

22. Signorini DF, Andrews PJ, Jones PA, et al. Adding insult to injury: the prognostic value of early secondary insults for survival after traumatic brain injury. J Neurol Neurosurg Psychiatr 1999;66:26-31. 
23. Walder AD, Yeoman PM, Turnbull A. The abbreviated injury scale as a predictor of outcome of severe head injury. Intensive Care Med 1995;21:606-9.

24. Choi SC, Narayan RK, Anderson RL, et al. Enhanced specificity of prognosis in severe head injury. J Neurosurg 1988;69:381-5.

25. Hsu MH, Li YC, Chiu WT, et al. Outcome prediction after moderate and severe head injury using an artificial neural network. Stud Health Technol Inform 2005;116:241-5.

26. Hukkelhoven CW, Steyerberg EW, Habbema JD, et al. Predicting outcome after traumatic brain injury: development and validation of a prognostic score based on admission characteristics. J Neurotrauma 2005;22:1025-39.

27. Murray GD, Butcher I, McHugh GS, et al. Multivariable prognostic analysis in traumatic brain injury: results from the IMPACT study. J Neurotrauma 2007;24:329-37.

28. Perel $\mathrm{P}$, Arango M, Clayton $\mathrm{T}$, et al. Predicting outcome after traumatic brain injury: practical prognostic models based on large cohort of international patients. BMJ 2008;336:425-9.

29. Turgeon AF, Lauzier F, Burns KE, et al. Determination of neurologic prognosis and clinical decision making in adult patients with severe traumatic brain injury: a survey of Canadian intensivists, neurosurgeons, and neurologists. Crit Care Med 2013;41:1086-93.

30. Turgeon AF, Lauzier F, Scales DC, et al. Practice variation in the management and treatment of increased intracranial pressure in severe traumatic brain injury in Canada: a Canadian multicentre retrospective cohort study [abstract]. Crit Care Med 2009;37:A435.

31. Jennett $B$, Bond MR. Assessment of outcome after severe brain damage. Lancet 1975;1:480-4.

32. Jennett B, Snoek J, Bond M, et al. Disability after severe head injury: observations on the use of the Glasgow outcome scale. J Neurol Neurosurg Psychiatr 1981;44:285-93.

33. Tyson SF, Thomas N, Vail A, et al. Recruiting to inpatient-based rehabilitation trials: lessons learned. Trials 2015;16:588.
34. Fletcher B, Gheorghe A, Moore D, et al. Improving the recruitment activity of clinicians in randomised controlled trials: a systematic review. BMJ Open 2012;2:e000496.

35. Dickson S, Logan J, Hagen S, et al. Reflecting on the methodological challenges of recruiting to a United Kingdom-wide, multi-centre, randomised controlled trial in gynaecology outpatient settings. Trials 2013;14:389.

36. Blanton S, Morris DM, Prettyman MG, et al. Lessons learned in participant recruitment and retention: the EXCITE trial. Phys Ther 2006;86:1520-33.

37. Gardner G, Gardner A, MacLellan L, et al. Reconceptualising the objectives of a pilot study for clinical research. Int J Nurs Stud 2003;40:719-24.

38. Mclntyre LA, Fergusson D, Cook DJ, et al. Fluid resuscitation in the management of early septic shock (FINESS): a randomized controlled feasibility trial. Can J Anaesth 2008;55:819-26.

39. Harrison DA, Prabhu G, Grieve R, et al. Risk Adjustment In Neurocritical care (RAIN) — prospective validation of risk prediction models for adult patients with acute traumatic brain injury to use to evaluate the optimum location and comparative costs of neurocritical care: a cohort study. Health Technol Assess 2013;17:vii-viii.

40. Andrews PJ, Sinclair HL, Rodriguez A, et al. Hypothermia for intracranial hypertension after traumatic brain injury. N Engl $J$ Med 2015;373:2403-12.

41. Wright DW, Yeatts SD, Silbergleit R, et al. Very early administration of progesterone for acute traumatic brain injury. $N$ Engl $J$ Med 2014;371:2457-66.

42. Potter JE, McKinley S, Delaney A. Research participants' opinions of delayed consent for a randomised controlled trial of glucose control in intensive care. Intensive Care Med 2013;39:472-80.

43. Gamble C, Nadel S, Snape D, et al. What parents of children who have received emergency care think about deferring consent in randomised trials of emergency treatments: postal survey. PLOS ONE 2012;7:e35982. 